\title{
DISTRIBUTION OF PARTICLE SIZES IN FOOD COMMINUTED BY HUMAN MASTICATION
}

\author{
I. W. Olthoff, A. van der Bilt, F. Bosman and H. H. Kleizen* \\ Departments of Gnathology and Dental Physics, School of Dentistry, State University of Utrecht, P.O. \\ Box 80,080, Utrecht, The Netherlands
}

\begin{abstract}
Summary-The masticatory performance of an individual may be quantified by describing the size distribution of particles of a comminuted test food as a function of the number of chewing strokes. A standardized sieving method and a standardized artificial test food (Optosil ".) were used to obtain reproducible results. Some measurements were performed using peanuts. The distribution of particle sizes of the comminuted food was adequately described by a Rosin-Rammler distribution function for the seven participating individuals which characterizes the size distribution by the median particle size $\left(x_{50}\right)$ and the broadness of the distribution $(b)$. The broadness variable $b$ hardly depends on the number of chewing strokes, and the differences in $b$ between the individuals are small. Considerable differences in the median particle sizes at a given number of chewing strokes were observed between the subjects, reflecting differences in efficiency of comminution of a test food. For all subjects, the median particle size decreased as a function of the number of chewing strokes $N$ according to the relation, $x_{50}=c \times N$ " Variables $c$ and $d$ characterized the efficiency of comminution by the subjects.
\end{abstract}

\section{INTRODUCTION}

In studies of the chewing performance, considerable attention has been given to such problems as the method of determining and analysing the particle sizes of the chewed food and the choice of the test food. Masticatory performance can be measured by determining an individual's capacity to grind or pulverize a test food. In the dental literature different requirements can be found for the procedures used to analyse the efficiency of comminution. Comminution of food has been determined by e.g. chemical analysis (Eichner, Hermann and Munk, 1954; Heath, 1982) and a colorimetric method. For comminuted food, consisting of particles with diameters between 0.1 and $10 \mathrm{~mm}$, comminution can be determined by measuring the particle size reduction achieved. In this size range test sieving has proved to be an appropriate method.

Since Gaudenz (1900) first introduced a sieving method in studies or chewing, a wide variety of such methods has been employed. Some workers have used test sieving w:th only one sieve. Manly and Braley (1950) definec the masticatory performance by the percentage of masticated peanuts which would pass a sieve with a fixed apcrturc. Others followed this procedure; for example, Yurkstas, (1951), Kapur and Soman (1964) Assha, Bhalla and Khanna (1971) and Rissin et al. (19\%8). Sieving mcthods using morc than one sieve were tmployed by Christiansen (1923) Dahlberg (1942), Parma (1952), Derksen, van Haeringen and Visser (1958), Helkimo (1978), Edlund and Lamm (1980) and Lucas and Luke (1983). These methods gave more detailed information on the distribution of particle sizes in the chewed

Department of Chemical Technology, Technical University of Delft, The Netherlands. food. However, the larger amount of data obtained in this way also complicates the description of the particle size distribution. One of our aims was to determine whether such detailed information on particle sizes can be expressed by a distribution function characterized by a few variables without losing essential information. If such a description is possible the large amount of data can be reduced to a few variables.

Both natural and artificial food have been used as test materials in experiments determining the masticatory performance (Manly and Braley, 1950; Edlund and Lamm, 1980). A natural test food has the advantage that it is normally consumed, so that subjects are accustomed to it. However, the consistency may vary due to seasonal and geographical influences. To avoid these variations in consistency, our experiments were performed with Optosil green (Bayer; Stock, 1980), a dental impression material used by Edlund and Lamm (1980). Both form and consistency can be easily reproduced. Some experiments were performed with peanuts in order to compare artificial and natural food.

\section{MATERIALS AND METHODS}

Seven individuals free of overt neuromuscular and mandibular joint disorders, participated. The subjects $A$ and $B$ had lost all molar teeth, whereas the other subjects $(C-G)$ had complete dentitions. Subject A had bad fitting dentures which caused pain during chewing.

Eight cubes of Optosil with an edge of $8 \mathrm{~mm}$, prepared according to instructions of the manufacturer (Bayer), were judged by the subjects as an adequate amount $(7.5 \mathrm{~g})$ for one mouthful (Lucas and Luke, 1984). The volume of this amount of test food was approx. $4 \mathrm{~cm}^{3}$. The taste of Optosil is more or less 
indifferent and at least caused no discomfort. The experiments using peanuts as a test food were performed on subjects $\mathrm{C}, \mathrm{E}$ and $\mathrm{F}$. The quantity of peanuts was chosen to match the volume of the Optosil portions.

\section{Experimental procedure}

During the experiments, the subjects were seated comfortably and upright in a dental chair, with head rest, in a quiet room. Instructions were given to chew in a habitual way. The number of chewing movements was counted by the examiner. After completion of the prefixed number of strokes, the chewed particles were expectorated into a cup. In order to obtain enough material for sieving, each test run consisted of 3 subruns in which 8 cubes of Optosil were offered. This whole procedure was repeated twice in order to reduce experimental scatter.

The food particles were washed with water and dried in an oven at $60^{\circ} \mathrm{C}$ for approx. $3 \mathrm{~h}$. Mass balances revealed weight losses of only 1 to 5 per cent for Optosil increasing with the number of chewing strokes $(10-100)$.

\section{Sieving procedure}

The sieving procedure is based upon internationally-accepted standard sieving methods (Allen, 1981). The sieves used accorded with the International Test Sieve Standard R 565. Wire sieves (ISO 3310-1) were selected because of their larger effective sieving area. The smallest sieve diameter available $(100 \mathrm{~mm})$ was selected in order to get the highest possible number of particles pro sieve aperture. Sieve apertures followed the R20-3 principal sizes implying a sieve aperture ratio of $2^{1 / 2}$. The sieve apertures of the 14 sieves used in our experiments were: $11.2,8.00,5.60,4.00,2.80,2.00,1.40,1.00$, $0.710,0.500,0.355,0.250,0.180$ and $0.125 \mathrm{~mm}$. The largest aperture was chosen such that each input food particle could pass through it. The sieves were arranged in stacks of maximal 10 sieves. The maximum time for stack sieving was set at $5 \mathrm{~min}$. After stack sieving, sieving was continued for a maximum time of 5 min with each individual sieve. The minimum time for treatment was defined when the rate of passage had dropped to less than 0.1 per cent of the weight on each sieve per minute. Reproducibility of the procedure was better than 1 per cent. The weight loss over the sieving was less than 1 per cent.

\section{Data analysis}

The weight of the particles on each sieve can be represented conveniently in a plot showing the cumulative weight percentages undersize as a function of the sieve aperture (= particle size). Data points plotted in this way can be seen in Fig. 1. The cumulative weight percentage undersize for a certain sieve aperture is defined as the percentage of the particles by weight that can pass that sieve. A logarithmic scale was chosen for the sieve apertures, because of the wide range in sieve apertures $(0.125-11.2 \mathrm{~mm})$, thus avoiding congestion of data points in the region of small sieve apertures.

In the literature, a number of empirical relations have been proposed to curve-fit the size distribution of industrial breakage products (Allen, 1981). The
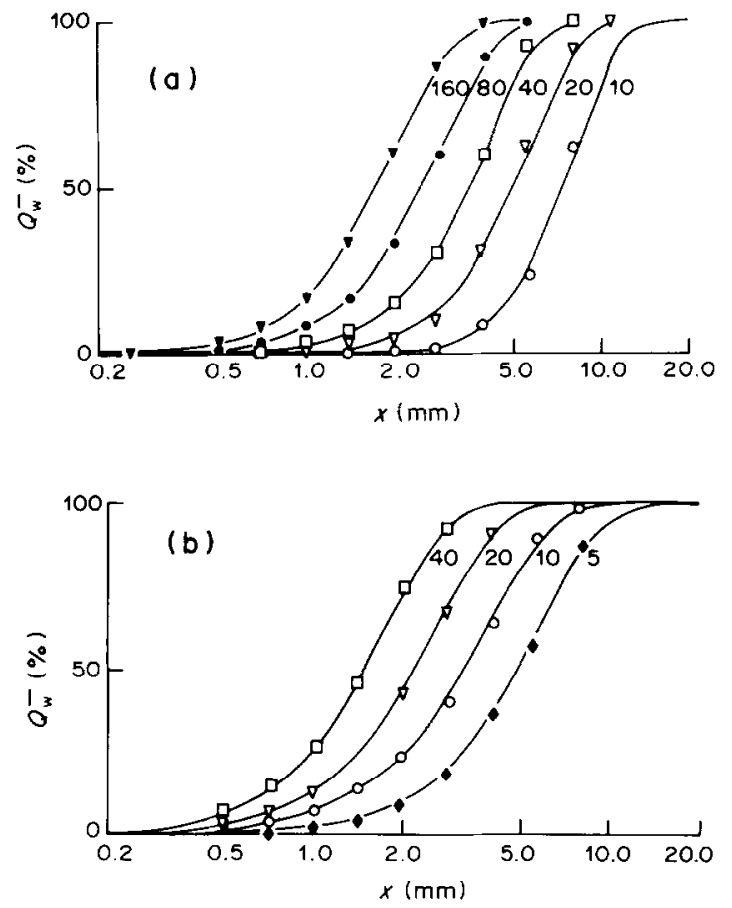

Fig. 1. Cumulative weight percentage undersize $Q_{w}^{-}$ (percentage) as a function of the logarithm of the sieve aperture $x(\mathrm{~mm})$ for individual $\mathrm{C}$ after various numbers of chewing strokes. (a) Optosil; (b) peanuts. Drawn lines are best fits through the data points according to equation (1).

Rosin-Rammler equation (Rosin and Rammler, 1933) has proved to give an adequate description of the particle-size distribution. To get a normalized distribution function the following transcription of the Rosin-Rammler equation was chosen:

$$
Q_{\mathrm{w}}^{-}=100\left\{1-\exp \left[-\left(x / x_{50}\right)^{h} \cdot \ln 2\right]\right\}
$$

where $Q_{w}^{-}$is the weight percentage of particles with a size smaller than $x$; the median $x_{50}$ is the aperture of a theoretical sieve through which 50 per cent of the weight can pass and $b$ is a variable indicating the broadness of the distribution $(0<b<\propto)$. Expression (1) should be corrected by a factor depending on $x_{50}, b$ and the maximum sieve aperture $x_{\mathrm{m}}$ to get a cumulative value of 1 for $x=x_{\mathrm{m}}$. However, since this correction is smaller than 1 per cent for all experimental conditions it is omitted for convenience. Increasing values of $b$ correspond to curves with steeper slopes and thus to distributions of particle sizes that are less broad. The variables $x_{50}$ and $b$ were determined by curve-fitting the data on equation (1) using a least-squares method.

\section{RESIJLTS}

Representative examples of particle size distributions as obtained for subject $\mathrm{C}$ after various numbers of chewing strokes are shown in Fig. la (Optosil) and Ib (peanuts). Data points represent sieve results, drawn lines the Rosin-Rammler curves which best fit the experimental points. The optimum values for $x_{50}$ and $b$ were obtained by minimizing the weighted sum 
of the squares of deviations of the data points of $Q_{\mathrm{w}}^{-}$ from the fitting function (Bevington, 1969). This minimized sum divided by the weighted average of the individual variances of the cumulative weights is characterized by the reduced chi-square probability distribution function $\chi_{v}^{2}$, where $v$ is the number of degrees of freedom. The error in the cumulative weights was approx. I per cent. The data points are well fitted by the function when the $\chi_{v}^{2}$-value determined for that fit is close to 1. Taking a confidence level of 95 per cent and having 9 data points the $\chi_{v}^{2}$-value should be smaller than 2.01 . The $\chi_{v}^{2}$-values obtained for the various fits ranged from 0.3 to 1.8 indicating that indeed the fitting function is a good approximation of the data for both Optosil and peanuts (Figs 1a and $1 b$ ). The accuracy of the determination of $x_{50}$ and $b$ was of the order of 2 per cent for all fits. Chewing an increasing number of times resulted in a shift of the Rosin-Rammler curves towards smaller particle sizes. The slopes of the curves did not charige much as a function of the number of chewing strokes. The shift towards smaller particle sizes as a function of the number of chewing strokes was more pronounced for the peanut results, indicating that peanuts are more easily comminuted than Optosil.

The cumulative weight percentage undersize of Optosil after 80 chewing strokes was plotted versus the particle size in Fig. 2 for each of the seven subjects. The data points were fitted successfully by the Rosin-Rammler equation. Considerale differences in the rnedian particle sizes, $x_{50}$ were observed for the subjects; the slopes of the curves were more or less the same. The particle size distribution cannot be described adequately by the Rossin-Rammler equation in the beginning of the chewing process. In that phase hardly any particle has been broken already, so that all particles have more or less the same size, resulting in a step-like distribution of sizes. However, good fits were obtained for all experiments on Optosil and peanuts whenever the median particle size $x_{50}$ had dropped to about 70 per cent of its starting value. This reduction in the median particle size was achieved after chewing at least 10 times on Optosil or 5 times on peanuts. However, subject A. needed more chewing strokes $(\geqslant 20)$ to obtain the desired reduction of the median

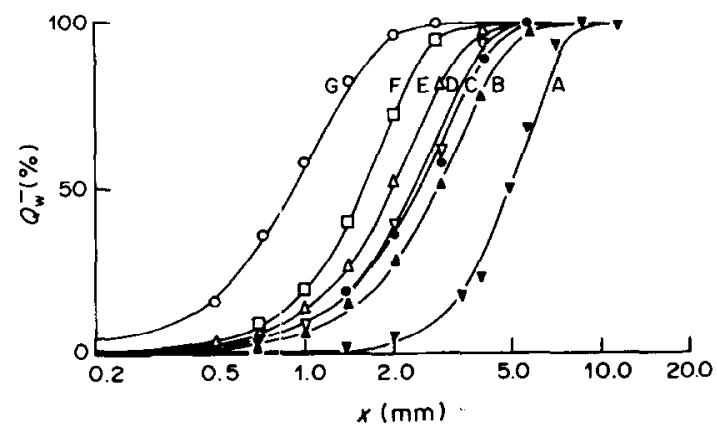

Fig. 2. Cumulative weight percentage undersize $Q_{w}^{-}$ (percentage) as a function of the logarithm of the sieve aperture $x(\mathrm{~mm})$ for seven individuals $(\mathrm{A}-\mathrm{G})$ after 80 chewing strokes (Optosil). Drawn lines are best fits through the data points according to equation (1).

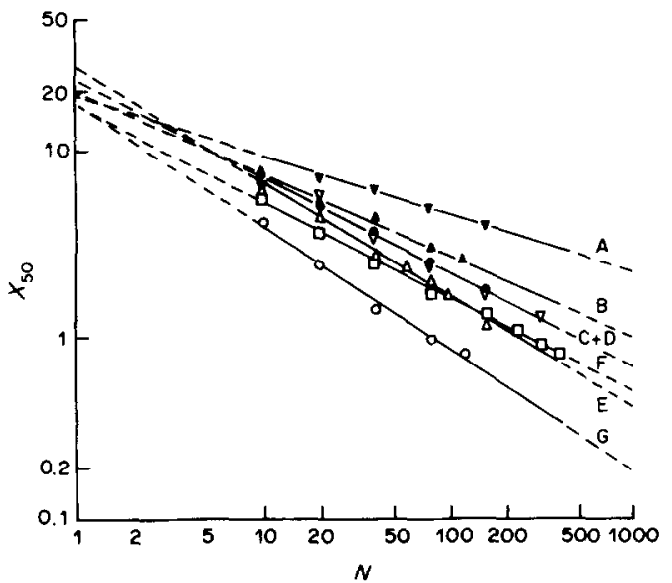

Fig. 3. Calculated values of the median $x_{50}(\mathrm{~mm})$ plotted versus the number of chewing strokes $N$ on a double logarithmic scale for seven subjects $(A-G)$ (Optosil). Drawn lines are best fits through the data points according to equation (2).

particle size of Optosil, so that the data point at $N=10$ was omitted for this subject.

The calculated values of $x_{50}$ for Optosil were plotted versus the number of chewing strokes $(N)$, on a double logarithmic scale in Fig. 3, for each of the seven subjects. On these scales a linear relation existed, so that the relationship between $x_{50}$ and $N$ is of the type:

$$
x_{50}=c \times N^{-d}
$$

where $c$ and $d$ are two variables describing the rate of the median particle size reduction as a function of the number of chewing strokes. Curve fitting yielded values for the reduced chi-square probability distribution function $\chi_{v}^{2}$ of the order of 1 for all subjects, indicating that the variation of data around the fitting function had the same magnitude as the accuracy of the data points $(\simeq 2 \%)$. The values of $c$ and $d$ determined from curve-fitting the data points are given in Table 1.

The values of the broadness variable $b$ for Optosil were plotted on a linear scale versus the logarithm of the number of chewing strokes in Fig. 4. The dependence of variable $b$ on $N$ was weak, though there was a definite tendency of decrease in the beginning $(N \leqslant 20)$ and of increase in the end of the chewing process $(N \geqslant 100)$ leading to U-shaped curves.

Table 1. Values and standard deviations of variables $c$ and $d$ of the relation between median $x_{50}$ and number of chewing strokes $N: x_{50}=c \times N^{-d}$

\begin{tabular}{ccccc}
\hline & $c$ & $d$ & $c$ & $d$ \\
Subject & \multicolumn{2}{c}{$\begin{array}{c}c \\
(\mathrm{~mm})\end{array}$} & & \multicolumn{2}{c}{$\begin{array}{c}\text { (Optosil) } \\
\text { (Peanuts) }\end{array}$} \\
\hline A & $19.2(0.7)$ & $0.32(0.03)$ & & \\
B & $21.4(1.3)$ & $0.46(0.05)$ & & \\
C & $24.6(0.4)$ & $0.52(0.01)$ & $16(5)$ & $0.6(0.1)$ \\
D & $23.6(1.3)$ & $0.52(0.04)$ & & \\
E & $26.1(0.9)$ & $0.61(0.03)$ & $15(5)$ & $0.6(0.1)$ \\
F & $16.8(0.6)$ & $0.51(0.02)$ & $14(5)$ & $0.6(0.1)$ \\
G & $17.2(1.2)$ & $0.66(0.06)$ & & \\
\hline
\end{tabular}




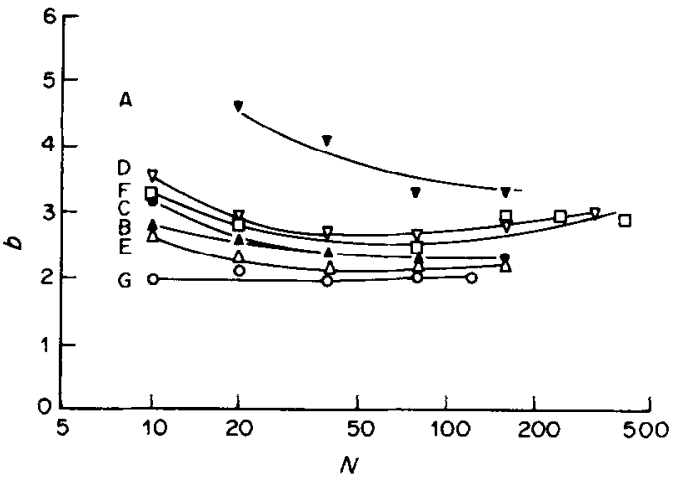

Fig. 4. Calculated values of the broadness variable $b$ as a function of the logarithm of the number of chewing strokes $N$ for seven subjects (A-G) for Optosil. Drawn lines are for visual aid only.

\section{DISCUSSION}

It is not possible to compare the results on masticatory performance obtained with the various experimental methods described in literature as no standardization in the employed methods exists. In order to enable such a comparison, both a standardized particle size analysis and a standardized test food must be used.

In order to make an estimate of the reproducibility within one test run these variables were determined three times on the same day (intra-test run reproducibility). This procedure was carried out on three different days with an interval of about two weeks (inter-test run reproducibility). These experiments were performed on four individuals $(C, D, E$, F) chewing 80 times on Optosil. The results are given in Table 2. Using analysis of variance with a significance level of 1 per cent no significant differences could be demonstrated for the values of $x_{50}$ or $b$ as obtained on differrent days. Thus the three series of three measurements can be combined to one group of nine measurements. Th,e mean and the standard deviation of these groups are also given in Table 2. The relative standard deviations of $x_{50}$ as well as $b$ are the order of 5 per cent for these subjects.
A quantitative description of the comminution of the test food during chewing can be given by determining the relation between the Rosin-Rammler variables and the number of chewing strokes. The variable $b$, indicating the broadness of the distribution of particle sizes, shows a dependence on the number of chewing strokes leading to U-shaped curves for all subjects. However, the increase of $b$ observed after a large number of chewing strokes $(N \geqslant 100)$ has not yet started for subject $A$. The values of $b$ are definitely different for the various subjects, though the differences are small.

A linear relation exists between the logarithm of the median particle size $x_{50}$ and the logarithm of the number of chewing strokes $N$ (equation 2) for all participating subjects (Fig. 3). The variables $c$ and $d$ of equation (2), together with their standard deviations as determined from regression analysis, are given in Table 1 for Optosil and peanuts. The variable $c$ defines the fictive median particle size after one chewing stroke. This value has only a theoretical meaning because the median particle size is not described by relation (2) after one chewing stroke. The variable $d$ (the slope when $x_{50}$ and $N$ are plotted on double logarithmic scales), gives information about the decrease of the median particle size per chewing stroke. The reproducibility of the peanut results is poor, as can be seen from the relatively large values for the standard deviations in the variables $c$ and $d$. Apparently, properties of the material, like consistency and geometry, are not constant for peanuts. Such factors are far more constant for Optosil leading to smaller standard deviations. It was tested whether the differences in the values of the two variables $c$ and $d$ observed for the various subjects are statistically significant. Using regression analysis, 95 per cent confidence regions for the regression lines in the double logarithmic plot were calculated. An overlap of the confidence regions of two regression lines for all values of $N$ between 10 and 160 chews indicates that no significant difference between these two lines exist. In this way, differences between the regression lines of all pairs of subjects were tested with a confidence level of 95 per cent. Significant differences were demonstrated for all pairs of subjects

Table 2. Values of the median particle size $x_{50}(\mathrm{~mm})$ and the broadness variable $b$ after 80 chewing strokes for the individuals C, D, E and F (Optosil)

\begin{tabular}{|c|c|c|c|c|c|c|c|c|}
\hline \multirow{2}{*}{$\begin{array}{l}\text { Session } \\
\text { number }\end{array}$} & \multicolumn{2}{|c|}{ C } & \multicolumn{2}{|c|}{ D } & \multicolumn{2}{|c|}{$E$} & \multicolumn{2}{|c|}{$\mathbf{F}$} \\
\hline & $x_{50}$ & $b$ & $x_{50}$ & $b$ & $x_{50}$ & $b$ & $x_{50}$ & $b$ \\
\hline \multirow{4}{*}{ I } & 2.27 & 2.69 & 2.17 & 2.70 & 1.94 & 2.44 & 1.65 & 2.46 \\
\hline & 2.27 & 2.44 & 2.05 & 2.70 & 1.96 & 2.28 & 1.66 & 2.33 \\
\hline & 2.44 & 2.57 & 2.09 & 2.74 & 2.14 & 2.29 & 1.66 & 2.39 \\
\hline & 2.33 & 2.29 & 2.43 & 2.71 & 2.00 & 2.21 & 1.65 & 2.42 \\
\hline \multirow[t]{3}{*}{ II } & 2.36 & 2.46 & 2.31 & 2.73 & 1.95 & 2.32 & 1.70 & 2.42 \\
\hline & 2.38 & 2.33 & 2.39 & 2.62 & 2.00 & 2.35 & 1.69 & 2.55 \\
\hline & 2.04 & 2.35 & 2.35 & 2.50 & 1.96 & 2.24 & 1.61 & 2.31 \\
\hline \multirow[t]{2}{*}{ III } & 2.24 & 2.25 & 2.06 & 2.60 & 1.98 & 2.23 & 1.67 & 2.25 \\
\hline & 2.15 & 2.38 & 2.09 & 2.57 & 1.92 & 2.31 & 1.58 & 2.30 \\
\hline Mean & 2.28 & 2.42 & 2.21 & 2.65 & 1.98 & 2.30 & 1.65 & 2.38 \\
\hline $\mathrm{SD}$ & 0.12 & 0.14 & 0.16 & 0.08 & 0.06 & 0.07 & 0.04 & 0.09 \\
\hline
\end{tabular}

Intra-test run reproducibility is obtained from the three values within a session and inter-test run reproducibility is obtained from the values of the three sessions. Mean values and standard deviations refer to nine measurements. 
except for the pair $C$ and $D$, when using Optosil as test food. The differences in the values of the two variables $c$ and $d$ observed for the various subjects when using Optosil indicate differences in masticatory performance. Using Optosil as a standardized test food and describing the particle sizes of the comminuted food with the Rosin-Rammler distribution function offers an opportunity to quantify the masticatory performance of an individual in a reproducible way.

Acknowledgement - We wish to thank Dr J. van Brakel, Dr H. W. van der Glas and Dr P. W. Lucas for their valuable discussions and for a critical reading of the manuscript.

\section{REFERENCES}

Allen T. (1981) Particle Size Measurement, 3rd edn. Chapman \& Hall, Londorl.

Asha, Bhalla L. R. and Khanna V. K. (1971) Evaluation of masticatory performance in canine protected occlusion. $J$. Ind. dent. Ass. 175-181.

Bevington P. R. (1969) Data Reduction and Error Analysis for the Physical Sciences. McGraw-Hill, New York.

Christiansen E. G. (19:23) Einige Untersuchungen über das Kauvermogen des natürlichen und des künstlichen Gebisses. Vjschr. Zahnhk. 39, 1-17.

Dahlberg B. (1942) The masticatory effect. Acta med. scand. $112,139$.

Derksen A. A. D., Haeringen W. van and Visser J. B. (1958) Het Kauwrendement. T. Tandheelk. 65, 381-426.

Edlund J. and Lamm C. J. (1980) Masticatory efficiency. $J$. oral Rehab. 7, 123-130.
Eichner K., Hermann D. and Munk P. (1954) Ein Beitrag zur Theorie und Praxis der totalen Prothese. Kaueffecktsprüfungen mit verschiedenen Verfahren bei natürlichen und künstlichen Gebisses. Dt. zahnärz. Z. 9, $1427-1435$.

Gaudenz J. U. (1900) Ueber die Zerkleinerung und Lösung von Nahrungsmitteln beim kauact. Arch. Hyg. 39, 230-25l.

Heath M. R. (1982) The effect of maximum biting force and bone loss upon masticatory function and dietary selection of the elderly. Int. dent. J. 32, 345-356.

Helkimo E., Carlsson G. E. and Helkimo M. (1978) Chewing efficiency and state of dentition. A methodologic study. Acta odont. scand. 36, 33-41.

Kapur K. and Soman S. D. (1964) Masticatory performance and efficiency in denture wearers. J. prosth. Dent. 14, 687-694.

Lucas P. W. and Luke D. A. (1983) Mcthods for analysing the breakdown of food in human mastication. Archs oral Biol. 28, 813-819.

Lucas P. W. and Luke D. A. (1984) Optimum mouthful for food comminution in human mastication. Archs oral Biol. 29, 205-210.

Manly R. S. and Braley L. C. (1950) Masticatory performance and efficiency. $J$. dent. Res. 29, 448-462.

Parma C. (1952) Die Kaufähigkeit bei Erkrankungen des Verdauungstraktus. Ost. Z. Stomat. 49, 643-652.

Rissin L., House J. E., Manly R. S. and Kapur K. K. (1978) Clinical comparison of masticatory performance and electromyographic activity of patients with complete dentures, overdentures and natural teeth. $J$. prosth. Dent. 39, 508-511.

Rosin P. and Rammler E. (1933) Gesetzmassigkeiten in der Kornzusammensetzung des Zementes. Zement 31, 427-433.

Yurkstas A. (1951) Compensation for inadequate mastication. Br. dent. J. 91, 261-262. 